\title{
Fractonic superfluids
}

\author{
Jian-Keng Yuan, ${ }^{1}$ Shuai A. Chen, ${ }^{2, *}$ and Peng $\mathrm{Ye}^{1, \dagger}$ \\ ${ }^{1}$ School of Physics, Sun Yat-sen University, Guangzhou 510275, China \\ ${ }^{2}$ Institute for Advanced Study, Tsinghua University, Beijing 100084, China
}

(Received 23 March 2020; accepted 15 May 2020; published 2 June 2020)

\begin{abstract}
We propose a superfluid phase of "many-fracton system" in which charge and total dipole moments are conserved quantities. In this work, both microscopic model and long-wavelength effective theory are analyzed. We start with a second quantized microscopic model and formulate the coherent-state path-integral representation. With repulsive interactions and positive chemical potential, we calculate various properties of the resulting superfluid state and make comparison with a conventional superfluid. We deduce a highly nonlinear Euler-Lagrange equation as well as two Noether currents. We also formulate time-dependent Gross-Pitaevskiitype equations that govern hydrodynamical behaviors. We study the classical ground-state wave function, the associated off-diagonal long range order (ODLRO), supercurrents, critical current, and unconventional topological vortices. At length scale much larger than coherence length $\xi_{\text {coh }}$, we derive the effective theory of our microscopic model. Based on the effective theory, we analyze gapless Goldstone modes and specific heat capacity at low temperatures as well as the fate of ODLRO against quantum fluctuations. Several future directions, e.g., numerical analysis of Gross-Pitaevskii equations, fermionic fractons, fractonic superconductors, and cold-atom experimental realization, are discussed.
\end{abstract}

DOI: 10.1103/PhysRevResearch.2.023267

\section{INTRODUCTION}

Liquid helium-4 [1,2] is a typical quantum many-boson system described by a Ginzburg-Landau theory. With interactions between bosons, superfluidity is established with formation of an off-diagonal long range order (ODLRO) [3] and emergence of gapless Goldstone modes. Vortex configurations, which tend to eliminate ODLRO, is topologically characterized by the winding number of the circulating supercurrent. Superfluid is also a simple demonstration on MerminWagner (MW) theorem which states that continuous symmetry cannot be spontaneously broken at any finite temperatures $(T)$ in one-dimensional (1D) and 2D systems. At zero temperature $T=0$, true ODLRO is unstable against quantum fluctuations unless the spatial dimension is no less than two. Experimentally, achievements have been made on a variety of physical properties of superfluidity; meanwhile, superfluids serve as a platform for different fields, e.g., condensed matter, nuclear physics and high-energy physics [4-16]. Especially, one may consider a symmetric phase formed by condensing symmetry defects in a superfluid or more general symmetrybreaking phases. By delicately designing degrees of freedom on symmetry defects, one may construct symmetry-protected topological phases (SPT) [12-15,17].

\footnotetext{
*s-chen16@mails.tsinghua.edu.cn

†yepeng5@mail.sysu.edu.cn
}

Published by the American Physical Society under the terms of the Creative Commons Attribution 4.0 International license. Further distribution of this work must maintain attribution to the author(s) and the published article's title, journal citation, and DOI.
In this paper, we propose an unconventional superfluid phase: fractonic superfluid, which was, surprisingly, motivated from seemingly uncorrelated line of thinking - strongly correlated topological phases of matter. Recently, there is an ongoing focus issue-fracton topological order [18-20] that supports topological excitations with restricted mobility. In contrast to the more "familiar" topological order such as the fractional quantum Hall effect, if one tries to move a fracton-a pointlike immobile excitation-then additional fractons have to be created nearby simultaneously. In other words, fractons are totally immobile. Tremendous progress has been made and vastly different research areas have been unexpectedly connected in the context of fractons, such as glassy dynamics, foliation theory, elasticity, dipole algebra, higher-rank global symmetry, many-body localization, stabilizer codes, duality, gravity, quantum spin liquid, and higherrank gauge theory, see, e.g., the review in Ref. [21] and Refs. [18-20,22-64].

While fractons are originally defined as pointlike excitations, one may also consider a many-fracton system-a quantum many-body system directly made of fractons. Suppose fractons are bosonic and simply represented by a scalar field $\phi$, one may ask: What kind of minimal microscopic quantum models can capture the property of immobility? Reference [43] recently proposed a non-Gaussian field theory by requiring that both total charge and total dipole moments be conserved, where the time derivative is second order and the momentum-dependent term involves $\phi$ of at least fourth order. This enhanced symmetry elegantly enforces the mobility restriction of single particles.

Alternatively, in this paper we consider a minimal model given by a second quantized microscopic Hamiltonian $\mathcal{H}$ that respects aforementioned symmetries. Then, the coherent-state 
path integral quantization sends $\mathcal{H}$ to $\mathcal{L}=i \phi^{*} \partial_{t} \phi-\mathcal{H}$ after a Wick rotation. It should be noted that the first-order derivative with respect to time in the Lagrangian $\mathcal{L}$ is very subtle. With this first-order derivative, one may legitimately interpret $\phi^{*} \phi$ as the particle number density, which is a common situation in nonrelativistic microscopic models in condensed matter physics and cold atom. Starting from this Lagrangian, we consider a weak repulsive interaction in a grand-canonical ensemble with positive chemical potential. The Euler-Lagrange equation of this theory is highly nonlinear, which is expected by observing that the Lagrangian as a functional of $\phi, \partial \phi$, and $\partial \partial \phi$ is intrinsically non-Gaussian. On the other hand, the Noether currents associated to the two conserved quantities are derived: charge current and dipole current. Using a hydrodynamic approach [1,2], we reformulate the Euler-Lagrange equation as hydrodynamic equations which help understand superfluidity.

We start with the normal state at $T=0$ with a negative chemical potential. When chemical potential is turned to positive value, the energy functional drops down to minima when $\phi$ belongs to plane-wave configurations, in contrast to conventional superfluid where $\phi$ of minima is exactly constant everywhere, i.e., momentum $\mathbf{k}=0$. This class of configurations with lowest energy constitutes the classical ground-state manifold of fractonic superfluid, and the corresponding timedependent Gross-Pitaevskii equations can be obtained in the presence of such exotic boson condensate. In a conventional superfluid, the charge (or more precisely, particle number) current serves as supercurrent. It can flow dissipationlessly as along as the current strength is below a critical value. The closed line integral of the supercurrent is topological in a sense that the numeric value of the integral only depends on how many vortices are enclosed by the closed line, resulting in a quantized value. Nevertheless, instead of charge current, in a fractonic superfluid, we have to identify a many-body current $\Xi$ as a supercurrent such that it is topological and can flow dissipationlessly. In fact, both closed line integrals of the two Noether currents mentioned above (charge and dipole current) turn out to be not topological. The corresponding quantized number monitored by $\oint \boldsymbol{\Xi}$ represents unconventional topological vortices that are expected to proliferate at critical points. Such a vortex shows interesting features and its dynamical behaviours deserve further investigation.

The Goldstone bosons associated to spontaneous broken symmetries in the many-fracton system are analyzed, whose dispersion relations give rise to exotic temperature dependence of specific heat capacity $c_{v}$ as long as ODLRO is assumed. Since quantum fluctuations are not treated seriously, ODLRO of classical ground states is self-consistently established, regardless of dimensions. For this purpose, one can integrate out massive amplitude fluctuations, resulting in an effective field theory for phase fluctuations or the gapless Goldstone bosons based on our microscopic many-fracton model. In the "isotropic case," the effective theory respects the Lifshitz spacetime symmetry and relates to nonrelativistic gravity studied before [65-67]. Once quantum fluctuations are taken into account, Goldstone bosons and ODLRO are ultimately unstable in $1 \mathrm{D}$ and $2 \mathrm{D}$. In $1 \mathrm{D}$, the correlation at long distance decays exponentially, which indicates a spectral gap is formed; in 2D, it decays in a power law. Compared to the conventional superfluid phase, all these dimensiondependence properties of ODLRO arise as a result of highly non-Gaussianality of many-fracton systems. A summary of comparison is given in Table I.

This paper is organized as follows. In Sec. II, we introduce a microscopic Hamiltonian in Eq. (1) and it conserves total dipole moments as well as a charge. We derive the EulerLagrange equation and Noether currents. A Gross-Pitaevskiitype equation is also formulated to govern hydrodynamic behavior. Section III starts with a Mexican-hat potential to determine a fractonic superfluid phase in any spatial dimensions with the ground-state wave functions in Eq. (34) from a hydrodynamic method. From the many-body current $\boldsymbol{\Xi}$ in Eq. (23) that appears as the supercurrent, we define a new vector field $\mathbf{U}$ in Eq. (48) whose vorticity turns out to be topological. In Sec. IV, we concentrate on quantum

TABLE I. Comparison between conventional and fractonic superfuild phases. For simplicity, in this table, only isotropic case $K_{i j}=\frac{1}{2} \kappa$ $(\kappa>0)$ of the microscopic model (1) is taken into account.

\begin{tabular}{lcc}
\hline \hline & Conventional superfluid & Fractonic superfluid \\
\hline Order parameter $\langle\hat{\Phi}(x)\rangle$ & $\sqrt{\rho_{0}} e^{i \theta_{0}}$ & $\sqrt{\rho_{0}} e^{i\left(\theta_{0}+\sum_{i} \beta_{i} x^{i}\right)}$ \\
Noether current & Charge current $J=\rho_{0} \nabla \theta$ & Charge current $J(20)$, dipole currents $\mathcal{D}^{(a)}(22)$ \\
Plane-wave dispersion & Dispersive & Dispersionless \\
Ground state & $\exp \left[\int d^{d} x \sqrt{\rho_{0}} e^{i \theta_{0}} \hat{\Phi}^{\dagger}(\mathbf{x})\right]|0\rangle$ & $\exp \left[\int d^{d} x \sqrt{\rho_{0}} e^{i\left(\theta_{0}+\sum_{i}^{d} \beta_{i} x^{i}\right)} \hat{\Phi}^{\dagger}(\mathbf{x})\right]|0\rangle$ \\
Topological number & $\ell=\oint_{C} \mathbf{v} \cdot d \mathbf{r}$ & $\ell=\oint_{C} \mathbf{U} \cdot d \mathbf{r}$ \\
Supercurrent & $\operatorname{Charge} \operatorname{current}$ & Many-body current $\mathbf{\Xi}$ in Eq. $(23)$ \\
Critical current & $|J|_{\max }=\frac{2 \sqrt{6}}{9} \sqrt{\frac{\mu^{3}}{g^{2} \kappa}}$ & $(\mathbf{\Xi})_{\max }=\frac{3 \sqrt{3 \kappa} \mu^{2}}{16 \sqrt{g^{3}}}$ in Eq. $(44)$ \\
Coherence length $\xi_{\text {coh }}$ & $2 \pi \sqrt{\kappa /\left(4 \rho_{0} g\right)}$ & $2 \pi \sqrt[4]{\kappa / 4 g}$ in Eq. (56) \\
Goldstone mode & $\omega \propto|\mathbf{k}|$ & $\omega \propto|\mathbf{k}|^{2}$ \\
Stable dimension at $T=0$ & $d>1$ & $d>2$ \\
\hline \hline
\end{tabular}


fluctuations or gapless Goldstone modes. With an effective theory for Goldtone modes, we calculate correlators of order parameters, and we give a temperature dependence of specific heat capacity. Last, Sec. V summarizes the main results and puts forward further perspectives. A general many-fracton model is discussed in the Appendix at the end of the paper.

\section{MICROSCOPIC MODEL}

In this section, we introduce a microscopic model and derive the Euler-Lagrange equation and the Noether currents, from which we recognize a Gross-Pitaevskii equation. All effective theory analysis in the remaining sections can be traced back to their microscopic origin introduced below.

\section{A. Model Hamiltonian}

In a nonrelativistic field theory, a single particle fails to propagate and may be eventually localized if its effective mass $M_{\text {eff }}$ is too large. In other words, the usual kinetic term $\frac{1}{2 M_{\mathrm{eff}}} \Phi^{\dagger}\left(-\nabla^{2}\right) \Phi$ vanishes. Nevertheless, it will be seen clear that mobility of bound-state excitations can be independent on single particle mobility. Let us consider a nonquadratic Hamiltonian. One realization is a model $H=\int d^{d} x \mathcal{H}\left(\hat{\Phi}^{\dagger}, \hat{\Phi}\right)$ in $d$ - (spatial) dimensional manifold $M$, where $\mathcal{H}$ reads

$$
\begin{aligned}
\mathcal{H}= & \sum_{i, j}^{d} K_{i j}\left(\hat{\Phi}^{\dagger} \partial_{i} \partial_{j} \hat{\Phi}^{\dagger}-\partial_{i} \hat{\Phi}^{\dagger} \partial_{j} \hat{\Phi}^{\dagger}\right)\left(\hat{\Phi} \partial_{i} \partial_{j} \hat{\Phi}-\partial_{i} \hat{\Phi} \partial_{j} \hat{\Phi}\right) \\
& +\sum_{i}^{d} G_{i}:\left(\partial_{i} \hat{\rho}\right)^{2}:+V\left(\hat{\Phi}^{\dagger}, \hat{\Phi}\right)
\end{aligned}
$$

where :: denotes the normal ordering. $\hat{\Phi}^{\dagger}(\mathbf{x})$ and $\hat{\Phi}(\mathbf{x})$ are bosonic creation and annihilation operators and $\hat{\rho}(\mathbf{x})=$ $\hat{\Phi}^{\dagger}(\mathbf{x}) \hat{\Phi}(\mathbf{x})$ is a density operator. These operators satisfy the standard communication relations

$$
\begin{gathered}
{\left[\hat{\Phi}(\mathbf{x}), \hat{\Phi}^{\dagger}(\mathbf{y})\right]=\delta^{d}(\mathbf{x}-\mathbf{y}),} \\
{[\hat{\Phi}(\mathbf{x}), \hat{\rho}(\mathbf{y})]=\hat{\Phi}(\mathbf{x}) \delta^{d}(\mathbf{x}-\mathbf{y}),} \\
{\left[\hat{\Phi}^{\dagger}(\mathbf{x}), \hat{\rho}(\mathbf{y})\right]=-\hat{\Phi}^{\dagger}(\mathbf{x}) \delta^{d}(\mathbf{x}-\mathbf{y}),}
\end{gathered}
$$

where $\mathbf{x}$ is a spatial coordinate and we neglect it in the following for symbol convenience. The potential $V\left(\hat{\Phi}^{\dagger}, \hat{\Phi}\right)$ incorporates interactions that do not involve any spatial gradient. We mainly consider the simplest form of $V\left(\hat{\Phi}^{\dagger}, \hat{\Phi}\right)$

$$
V\left(\hat{\Phi}^{\dagger}, \hat{\Phi}\right)=-\mu \hat{\Phi}^{\dagger} \hat{\Phi}+\frac{g}{2} \hat{\Phi}^{\dagger} \hat{\Phi}^{\dagger} \hat{\Phi} \hat{\Phi},
$$

where $\mu$ is the chemical potential and $g>0$ describes onsite repulsive interaction. Hereafter, no Einstein summation rule is assumed. The coupling constants $K_{i j}>0$ and $G_{i} \geqslant 0$ ensure a lower bound for a physically acceptable Hamiltonian $\mathcal{H}$. The $G_{i}$ term is also a potential term. Besides, no rotational invariance is assumed since anisotropy of $K_{i j}$ is allowed. The Hamiltonian in Eq. (1) is possible to be realized in cold atomic gas subjected to an optical lattice by simulating the correlated hopping of two bosons [68,69]. Hamiltonian $\mathcal{H}$ obeys a conservation law of total dipole moments as well as a global $U(1)$ symmetry. So we have two types of conserved quantities. One is the global $U(1)$ charge $\hat{Q}=\int d^{d} \mathbf{x} \hat{\rho}$ and the others are the total dipole moments of $d$ components $\hat{Q}^{(a)}=\int d^{d} \mathbf{x} \hat{\rho} x^{a}, a=1,2, \ldots, d$, where the integral is over the $d$-dimensional spatial manifold $M$. We denote a group generated by both $\hat{Q}$ and $\hat{Q}^{(a)}(a=1,2, \ldots, d)$ as $\mathcal{G}$ [70]. We denote a subgroup with a single generator $Q^{(a)}$ as $U(1)^{a}$. An element $U=\exp \left[-i\left(\lambda \hat{Q}+\sum_{a} \lambda_{a} \hat{Q}^{(a)}\right)\right]$ in $\mathcal{G}$ leads to a transformation of the field $\phi$ as $\phi^{\prime}=U \phi U^{\dagger}=\phi e^{i\left(\lambda+\sum_{a} \lambda_{a} x^{a}\right)}$ with $d+1$ real parameters $\lambda$ and $\lambda_{a}(a=1, \ldots, d)$. The group $\mathcal{G}$ is not an internal symmetry because $\hat{Q}^{(a)}$ does not commute with the translational or rotational symmetry.

By performing the coherent-state path integral quantization, we can construct a partition function $\mathcal{Z}=$ $\int \mathcal{D} \phi \mathcal{D} \phi^{*} e^{i \int d^{d} \mathbf{x} d t \mathcal{L}}$ with Lagrangian $\mathcal{L}$ from $\mathcal{H}$ in Eq. (1) as

$$
\mathcal{L}=i \phi^{*} \partial_{t} \phi-\mathcal{H}\left(\phi^{*}, \phi\right),
$$

where $\phi(\mathbf{x}, t)$ is the eigenvalue of annihilation operator $\hat{\Phi}(\mathbf{x})$ on a coherent state

$$
\hat{\Phi}(\mathbf{x})|\phi(\mathbf{x}, t)\rangle=\phi(\mathbf{x}, t)|\phi(\mathbf{x}, t)\rangle
$$

and $\phi^{*}(\mathbf{x}, t)$ is its complex conjugate. It should be noted that a Wick rotation has been applied from imaginary time to real time, which is convenient for the physics of zero temperature. The subtle "first-order time derivative" term in Eq. (6) is ultimately determined by Hamiltonian (1) and commutation relations listed in Eqs. (2), (3), and (4), which can be verified by performing the standard canonical quantization. We regard Eqs. (1) and (6) as the microscopic model of fractonic superfluids.

\section{B. Euler-Lagrange equation and Noether theorem}

The Noether theorem states that a classical action that respects a continuous symmetry is associated to a conserved charge. A continuity equation can be deduced from the action. Below we will derive the Euler-Lagrange equation as well as Noether currents from the Noether theorem. Due to the non-Gaussian nature of the microscopic model, the derivation below will demonstrate several exotic features that do not appear in usual Gaussian models.

First, we derive the Euler-Lagrange equation. Generally the stationary condition of an action $S=$ $\int d^{d} \mathbf{x} d t \mathcal{L}\left[\partial_{t} \phi, \partial_{i} \phi, \partial_{i} \partial_{j} \phi, \phi\right]$ meets the Euler-Lagrange equation. Here for the notational convenience, we do not explicitly show the dependence on $\phi^{*}$ and its derivative terms in $\mathcal{L}$. A variation $\delta \phi$ leads to $\delta S$

$$
\begin{aligned}
\delta S= & \int d^{d} \mathbf{x} d t \delta \mathcal{L}\left[\partial_{t} \phi, \partial_{i} \phi, \partial_{i} \partial_{j} \phi, \phi\right] \\
= & \int d^{d} \mathbf{x} d t\left[\frac{\delta \mathcal{L}}{\delta \phi}-\partial_{t} \frac{\delta \mathcal{L}}{\delta \partial_{t} \phi}-\sum_{i}^{d} \partial_{i} \frac{\delta \mathcal{L}}{\delta \partial_{i} \phi}\right. \\
& \left.+\sum_{i, j}^{d} \partial_{i} \partial_{j} \frac{\delta \mathcal{L}}{\delta \partial_{i} \partial_{j} \phi} \delta \phi\right]+ \text { c.c. }
\end{aligned}
$$

Here the variation does not depend on space-time coordinates, $\delta \partial_{i} \phi=\partial_{i} \delta \phi, \delta \partial_{i} \partial_{j} \phi=\partial_{i} \partial_{j} \delta \phi$, etc., and c.c. means complex conjugate. 
To a surface term, vanishing of Eq. (8) requires the EulerLagrange equation,

$$
\partial_{t} \frac{\delta \mathcal{L}}{\delta \partial_{t} \phi}=\frac{\delta \mathcal{L}}{\delta \phi}-\sum_{i}^{d} \partial_{i} \frac{\delta \mathcal{L}}{\delta \partial_{i} \phi}+\sum_{i, j}^{d} \partial_{i} \partial_{j} \frac{\delta \mathcal{L}}{\delta \partial_{i} \partial_{j} \phi}
$$

One remark is that we take $\partial_{i} \partial_{j} \phi$ and $\partial_{j} \partial_{i} \phi$ as different variables if $i \neq j$ during variational processes,

$$
\frac{\delta \partial_{i} \partial_{j} \phi(\mathbf{x}, t)}{\delta \partial_{m} \partial_{n} \phi\left(\mathbf{x}^{\prime}, t^{\prime}\right)}=\delta_{i m} \delta_{j n} \delta\left(\mathbf{x}-\mathbf{x}^{\prime}\right) \delta\left(t-t^{\prime}\right) .
$$

In sharp contrast to the usual Euler-Lagrange equations, there are three terms in the right-hand side of Eq. (9) where the third term arises from the non-Gaussionality. Plugging Eq. (6) into Eq. (9) renders

$$
\begin{aligned}
i \partial_{t} \phi= & \sum_{i, j}^{d} K_{i j} \partial_{i} \partial_{j}\left[\phi^{*}\left(\phi \partial_{i} \partial_{j} \phi-\partial_{i} \phi \partial_{j} \phi\right)\right] \\
& +2 K_{i j} \partial_{i}\left[\partial_{j} \phi^{*}\left(\phi \partial_{i} \partial_{j} \phi-\partial_{i} \phi \partial_{j} \phi\right)\right] \\
& +K_{i j} \partial_{i} \partial_{j} \phi^{*}\left(\phi \partial_{i} \partial_{j} \phi-\partial_{i} \phi \partial_{j} \phi\right) \\
& -\sum_{i}^{d} 2 G_{i} \partial_{i}^{2} \rho \phi-\mu \phi+g \rho \phi
\end{aligned}
$$

where we have considered a Mexican-hat potential in Eq. (6):

$$
V(\phi)=-\mu|\phi|^{2}+\frac{g}{2}|\phi|^{4} .
$$

It is the path-integral representation of the operator form $V\left(\hat{\Phi}^{\dagger}, \hat{\Phi}\right)$ in Eq. (5).

Solving Eq. (11) in a brute-force manner, both numerically and analytically, is not easy due to its high nonlinearity. Nevertheless, one may quickly verify the existence of immobile fractons just by taking a plane-wave ansatz,

$$
\phi=\mathscr{N} \exp (i \omega t-i \mathbf{k} \cdot \mathbf{x}),
$$

where $\mathscr{N}$ is a proper normalization factor. The flat dispersion relation $\omega=0$ indicates that a single particle is nonpropagating. Such kinds of particles with fully restricted mobility are dubbed fracton in the literature of fracton topological order.

Now we are in a position to calculate the Noether currents associated with the two conserved quantities. We consider on-shell variations where fields $\phi$ and $\phi^{*}$ are constrained to satisfy Euler-Lagrange equations while the variations $\delta \phi$ and $\delta \phi^{*}$ are arbitrary. A symmetry transformation

$$
\phi \rightarrow \phi^{\prime}=\phi+\delta \phi=\phi+\alpha F(\phi)
$$

has a parameter $\alpha$ that is independent of space-time coordinates and keeps the Euler-Lagrange equation invariant while it does not involve changes in the coordinates in any way. The Noether theorem states that even when $\alpha$ depends on coordinates $\alpha=\alpha(\mathbf{x}, t)$, the variation action $\delta S=\int d^{d} \mathbf{x} d t \mathcal{L}\left[\partial_{t} \phi^{\prime}, \partial_{i} \phi^{\prime}, \partial_{i} \partial_{j} \phi^{\prime}, \phi\right]-\mathcal{L}\left[\partial_{t} \phi, \partial_{i} \phi, \partial_{i} \partial_{j} \phi, \phi\right]$ should also vanish,

$$
\begin{aligned}
\delta S & =\int d^{d} \mathbf{x} d t \frac{\delta \mathcal{L}}{\delta \partial_{t} \phi} \delta \partial_{t} \phi+\sum_{i}^{d} \frac{\delta \mathcal{L}}{\delta \partial_{i} \phi} \delta \partial_{i} \phi+\sum_{i, j}^{d} \frac{\delta \mathcal{L}}{\delta \partial_{i} \partial_{j} \phi} \delta \partial_{i} \partial_{j} \phi+\frac{\delta \mathcal{L}}{\delta \phi} \delta \phi \\
& =\int d^{d} \mathbf{x} d t(-\alpha) \partial_{t}\left(\frac{\delta \mathcal{L}}{\delta \partial_{t} \phi} F\right)-\sum_{i}^{d} \alpha\left[\partial_{i}\left(\frac{\delta \mathcal{L}}{\delta \partial_{i} \phi} F\right)+\sum_{i, j}^{d} \partial_{i}\left(\frac{\delta \mathcal{L}}{\delta \partial_{i} \partial_{j} \phi} \partial_{j} F+\frac{\delta \mathcal{L}}{\delta \partial_{j} \partial_{i} \phi} \partial_{j} F\right)-\sum_{i, j}^{d} \partial_{i} \partial_{j}\left(\frac{\delta \mathcal{L}}{\delta \partial_{i} \partial_{j} \phi} F\right)\right],
\end{aligned}
$$

where the Euler-Lagrange equation in Eq. (9) is applied. The variation $\delta S$ appears as an integral over a total derivative

$$
\delta S=\alpha \int d^{d} \mathbf{x} d t\left(\partial_{t} \rho+\sum_{i}^{d} \partial_{i} J_{i}\right) .
$$

We arrive at conserved charge $Q$ and current densities $J_{i}$

$$
\begin{aligned}
Q & =-\int d^{d} \mathbf{x} \frac{\delta \mathcal{L}}{\delta \partial_{t} \phi} F+\text { c.c. }=\int d^{d} x \rho \\
J_{i} & =-\left(\frac{\delta \mathcal{L}}{\delta \partial_{i} \phi}-\sum_{j}^{d} \partial_{j} \frac{\delta \mathcal{L}}{\delta \partial_{i} \partial_{j} \phi}\right) F-\sum_{j}^{d} \frac{\delta \mathcal{L}}{\delta \partial_{i} \partial_{j} \phi} \partial_{j} F+\text { c.c. }
\end{aligned}
$$

and the conservation law

$$
\partial_{t} \rho+\sum_{i}^{d} \partial_{i} J_{i}=0
$$

Back to our model in Eq. (6), for a global $U(1)$ symmetry, we take $F(\phi)=i \phi$ and $F(\phi)=i x^{a} \phi$ for $U(1)^{(a)}$ and we can obtain charge and current densities,

$$
Q=\int d^{d} \mathbf{x} \phi^{*} \phi
$$

$$
\begin{gathered}
J_{i}=i \sum_{j}^{d} K_{i j} \partial_{j}\left[\phi^{* 2}\left(\phi \partial_{i} \partial_{j} \phi-\partial_{i} \phi \partial_{j} \phi\right)-\text { c.c. }\right], \\
Q^{(a)}=\int d^{d} \mathbf{x} x^{a} \phi^{*} \phi
\end{gathered}
$$

$$
\mathcal{D}_{i}^{(a)}=i \sum_{j}^{d} K_{i j} x^{a} \partial_{j}\left[\phi^{* 2}\left(\phi \partial_{i} \partial_{j} \phi-\partial_{i} \phi \partial_{j} \phi\right)-\text { c.c. }\right]
$$

$$
-i \sum_{j}^{d} K_{i j} \delta_{a}^{j}\left[\phi^{* 2}\left(\phi \partial_{i} \partial_{j} \phi-\partial_{i} \phi \partial_{j} \phi\right)-\text { c.c. }\right] .
$$


Therefore, we have two types of spatial currents: $J_{i}$ and $\mathcal{D}_{i}^{(a)}$. Nevertheless, they are not totally independent. The first term in current $\mathcal{D}_{i}^{(a)}$ in Eq. (22) that equals $x^{a} J_{i}$ comes from motions of each single particle at $\mathbf{x}$ with current $J_{i}$ and the extra term comes from the pure effect during many-body hopping processes. It motivates us to isolate the many-body current $\mathbf{\Xi}$,

$$
\Xi_{i a}=x^{a} J_{i}-\mathcal{D}_{i}^{(a)} .
$$

The many-body current $\boldsymbol{\Xi}$ is symmetric under its index and it has relation with charge current $J_{i}=\sum_{a=1}^{d} \partial_{a} \Xi_{i a}$. This relation implies a generalized conversation law $\partial_{t} \rho+\partial_{i} \partial_{a} \Xi_{i a}=$ 0 . As we will see in Sec. III that the current $\boldsymbol{\Xi}$ plays a vital role.

\section{Time-dependent Gross-Pitaevskii-type equations}

Below we will deduce equations that govern hydrodynamic behaviors of the superfluid, which are summarized as a timedependent Gross-Pitaevskii equation set. We now rewrite Eq. (11)

$$
i \partial_{t} \phi=\hat{H} \phi,
$$

where $\hat{H}$ behaves as a single-particle Hamiltonian that reads

$$
\begin{aligned}
\hat{H}= & \sum_{i, j}^{d} K_{i j} \partial_{i} \partial_{j}\left[\phi^{*}\left(-\partial_{i} \phi \partial_{j}+\phi \partial_{i} \partial_{j}\right)\right] \\
& +2 K_{i j} \partial_{i}\left[\partial_{j} \phi^{*}\left(\phi \partial_{i} \partial_{j}-\partial_{i} \phi \partial_{j}\right)\right] \\
& +K_{i j} \partial_{i} \partial_{j} \phi^{*}\left(\phi \partial_{i} \partial_{j}-\partial_{i} \phi \partial_{j}\right) \\
& -\sum_{i}^{d} 2 G_{i} \partial_{i}^{2} \rho-\mu+g \rho .
\end{aligned}
$$

Equation (24) has a similar form as a time-dependent GrossPitaevskii equation in a conventional superfluid phase where $g$ characterizes a hardcore interaction. Differently, the kinetic term is nonlinear due to refinement from the symmetry group $\mathcal{G}$. One way to understand Eq. (24) is to derive a hydrodynamic equation by decomposing $\phi=\sqrt{\rho} e^{i \theta}$ where the real fields $\rho$ and $\theta$ are density and phase operators, respectively. So the Gross-Pitaevskii equation is equivalent to two partial derivative equations,

$$
\begin{gathered}
\frac{\partial \rho}{\partial t}=2 \sum_{i, j}^{d} K_{i j} \partial_{i} \partial_{j}\left(\rho^{2} \partial_{i} \partial_{j} \theta\right) \\
\frac{\partial \theta}{\partial t}=-\frac{1}{2 \rho^{3}} \sum_{i, j}^{d} K_{i j}\left[\left(\partial_{i} \rho \partial_{j} \rho\right)^{2}-2 \rho \partial_{i} \rho \partial_{j} \rho \partial_{i} \partial_{j} \rho\right] \\
-\frac{1}{2 \rho} \sum_{i, j}^{d} K_{i j}\left[\left(\partial_{i} \partial_{j} \rho\right)^{2}-\partial_{i}^{2} \rho \partial_{j}^{2} \rho\right] \\
-\frac{1}{2} \sum_{i, j}^{d} K_{i j}\left[4 \rho\left(\partial_{i} \partial_{j} \theta\right)^{2}+\partial_{i}^{2} \partial_{j}^{2} \rho\right] \\
+\sum_{i}^{d} G_{i} \partial_{i}^{2} \rho+\mu-g \rho .
\end{gathered}
$$

Equation (26) is a continuity equation and the dynamics of $\theta$ is very complicated. The solution towards Eqs. (26) and (27) resembles a fluid with conserved dipole moments.

The hydrodynamic velocity $v_{i}$ is defined as

$$
J_{i}=\rho v_{i},
$$

where $\rho$ is the charge density. From $J_{i}$ in Eq. (20), we find that

$$
v_{i}=-\sum_{j}^{d} 2 K_{i j}\left(2 \partial_{j} \rho \partial_{i} \partial_{j} \theta+\rho \partial_{i} \partial_{j}^{2} \theta\right) .
$$

The "velocity" $v_{i}^{(a)}$ with a relation $\mathcal{D}_{i}^{(a)}=\rho v_{i}^{(a)}$ can also be deduced from Eq. (22) as

$$
v_{i}^{(a)}=2 K_{i a} \rho \partial_{i} \partial_{a} \theta+v_{i} x^{a} .
$$

It is easy to extract two continuity equations

$$
\begin{gathered}
\frac{\partial \rho}{\partial t}+\sum_{i}^{d} \partial_{i}\left(\rho v_{i}\right)=0, \\
\frac{\partial \rho^{(a)}}{\partial t}+\sum_{i}^{d} \partial_{i}\left(\rho v_{i}^{(a)}\right)=0 .
\end{gathered}
$$

Numerical simulations to Eqs. (26) and (27) may show interesting features, which can help us get insight into the GP equation in Eq. (24), and it deserves future investigations. Before moving to next section, we should emphasize that all equations, currents, and charges are not specified to a certain phase of the microscopic model. In the next section, we will focus on the superfluid phase.

\section{FRACTONIC SUPERFLUIDITY}

We have discussed basic properties like Noether currents and Gross-Pitaevskii equation in a many-fracton model in Eq. (1). The main feature is its non-Gaussian form resulting from a dipole-moment conservation symmetry $\mathcal{G}$. In this section, we discuss in details the fractonic superfluidity arising from our microscopic model.

\section{A. ODLRO and order parameter}

Superfluidity can occur in a conventional bosonic system with a potential $V(\phi)$ in Eq. (12). In this section, we consider a superfluid phase in a fracton system in the microscopic model (1).

Classically, the energy density $\mathcal{E}$ for the steady system in Eq. (1) has the form as

$$
\begin{aligned}
\mathcal{E}= & \sum_{i, j}^{d} K_{i j}\left|\phi \partial_{i} \partial_{j} \phi-\partial_{i} \phi \partial_{j} \phi\right|^{2} \\
& +\sum_{i}^{d} G_{i}\left(\partial_{i} \rho\right)^{2}-\mu|\phi|^{2}+\frac{g}{2}|\phi|^{4}
\end{aligned}
$$

and the field configuration $\phi$ at its minimum depends on the chemical potential. If $\mu<0$, then the potential $V(\phi)$ has a minimal value at $\rho=0$. It is a normal phase. If $\mu>0$, then the potential $V(\phi)$ reaches a minimal value at $|\phi|=\sqrt{\rho_{0}} \equiv$ $\sqrt{\frac{\mu}{g}}$. The vacuum now possesses a finite particle density and 
thus a large number of degeneracies. In the second quantization language, the ground-state manifold can be represented with a creation operator $\hat{\Phi}^{\dagger}$ along with phase parameters $\theta_{0}$ and $\beta_{i}(i=1, \ldots, d)$,

$$
\left|\mathrm{GS}_{\beta_{i}}^{\theta_{0}}\right\rangle=\bigotimes_{\mathbf{x}}\left|\mathrm{GS}_{\beta_{i}}^{\theta_{0}}\right\rangle_{\mathbf{x}}
$$

where $\left|G S_{\beta_{i}}^{\theta_{0}}\right\rangle_{\mathbf{x}}$ describes particles at position $\mathbf{x}$,

$$
\left|\mathrm{GS}_{\beta_{i}}^{\theta_{0}}\right\rangle_{\mathbf{x}}=\frac{1}{C} \exp \left[\sqrt{\rho_{0}} e^{i\left(\theta_{0}+\sum_{i}^{d} \beta_{i} x^{i}\right)} \Phi^{\dagger}(\mathbf{x})\right]|0\rangle
$$

with $C=e^{\frac{1}{2} \rho_{0}}$ as the normalization factor. For two such ground states $\left|\mathrm{GS}_{\beta_{i}}^{\theta_{0}}\right\rangle$ and $\left|\mathrm{GS}_{\beta_{i}^{\prime}}^{\theta_{0}^{\prime}}\right\rangle$ with $\Delta \theta_{0}=\theta_{0}-\theta_{0}^{\prime}, \Delta \beta_{i}=$ $\beta_{i}-\beta_{i}^{\prime}$, from their inner product,

$$
\begin{aligned}
& \left|\left\langle\mathrm{GS}_{\beta_{i}^{\prime}}^{\theta_{0}^{\prime}} \mid \mathrm{GS}_{\beta_{i}}^{\theta_{0}}\right\rangle\right|^{2} \\
& \quad=\left\{\begin{array}{lll}
\exp \left(-2 \rho_{0} V\right) & \text { if } \Delta \beta_{i} \neq 0 \quad \exists i \\
\exp \left[-2 \rho_{0} V\left(1-\cos \Delta \theta_{0}\right)\right] & \text { if } \Delta \beta_{i}=0 \quad \forall i
\end{array},\right.
\end{aligned}
$$

we can conclude that they are orthogonal in the thermodynamic limit $V \rightarrow \infty$, where $V$ is the volume of the spatial manifold $M$. The ground state in Eq. (34) comprises equalweight superposition over all possible numbers of particles that is modulated by a phase factor and it characterizes condensation of a macroscopically large number of particles at a state with momentum $\mathbf{k}=\left(\beta_{1}, \ldots, \beta_{d}\right)$ by observing $\left|\mathrm{GS}_{\beta_{i}}^{\theta_{0}}\right\rangle=\exp \left[\sqrt{\rho_{0}} e^{i \theta_{0}} \hat{\Phi}^{\dagger}(\mathbf{k})\right]|0\rangle$, where $\hat{\Phi}^{\dagger}(\mathbf{k})$ is the Fourier transformation of $\hat{\Phi}^{\dagger}(\mathbf{x})$. We call state $\left|\mathrm{GS}_{\beta_{i}}^{\theta_{0}}\right\rangle$ in Eq. (34) as a fractonic superfluid phase. The most significant feature of the state (34) is the formation of an ODLRO. If we calculate the correlation function in the classical level, then

$$
C(\mathbf{x})=\left\langle\mathrm{GS}_{\beta_{i}}^{\theta_{0}}\left|\hat{\Phi}^{\dagger}(\mathbf{x}) \hat{\Phi}(0)\right| \mathrm{GS}_{\beta_{i}}^{\theta_{0}}\right\rangle=\rho_{0} e^{-i\left(\sum_{i}^{d} \beta_{i} x^{i}\right)},
$$

whose amplitude does not decay at large distances. Equivalently, an order parameter can be determined with finite expectation value on the ground state,

$$
\langle\hat{\Phi}(\mathbf{x})\rangle=\left\langle\mathrm{GS}_{\beta_{i}}^{\theta_{0}}|\hat{\Phi}(\mathbf{x})| \mathrm{GS}_{\beta_{i}}^{\theta_{0}}\right\rangle=\sqrt{\rho_{0}} e^{i\left(\theta_{0}+\sum_{i} \beta_{i} x^{i}\right)} .
$$

\section{B. Supercurrent and its critical value}

Currents can suppress superfluidity. In a conventional superfluid phase, frictionless charge current can exist if superfluidity is not totally destroyed. In other words, superfluidity can survive as long as the system has a finite order parameter or particle density $\rho_{0}$ for the minimal total energy. We can expect a critical current as the maximum one that a conventional superfluid phase can sustain. Similar discussion can be applied to a fractonic superfluid phase. Here we investigate the critical current in a fractonic superfluid phase in an isotropic case $K_{i j}=\frac{1}{2} \kappa$.

The ground state in Eq. (34) can be considered as the one that minimizes the energy density $\mathcal{E}$ in Eq. (33) under a specified boundary condition on the net phase change. For example, a wave function $\left|\mathrm{GS}_{\beta_{i}}^{\theta_{0}}\right\rangle$ minimizes $\mathcal{E}$ in Eq. (33) under the boundary conditions

$$
\begin{aligned}
\Delta_{i} \theta & =\beta_{i} L \quad i=1, \ldots, d, \\
\Delta_{i} \partial_{j} \theta & =0 \quad i, j=1, \ldots, d,
\end{aligned}
$$

where $\Delta_{i} \theta\left(\Delta_{i} \partial_{j} \theta\right)$ is the net difference of field $\theta\left(\partial_{j} \theta\right)$ along $x^{i}$ direction with $L$ as the system size. A possible suppressing factor is equivalent to twisting the boundary conditions in Eqs. (39a) and (39b). For example, the net change of $\partial_{j} \theta$ can be twisted to a finite value, which will invalidate the form of field $\theta=\theta_{0}+\sum_{i=1}^{d} \beta_{i} x^{i}$ in Eq. (34). Our aim is to determine the order parameter and currents under a twisted boundary condition. Minimizing the energy density $\mathcal{E}$ in Eq. (33) requires uniformity of $\left|\nabla^{2} \theta(x)\right|$ and we denote $v=\left|\nabla^{2} \theta(x)\right|$. Based on it, in a superfluid phase with $\mu>0, \mathcal{E}$ in Eq. (33) can be written as

$$
\mathcal{E}=\frac{1}{2} \kappa \rho^{2}|\nu|^{2}-\mu \rho+\frac{g}{2} \rho^{2},
$$

where $\rho$ is particle density of condensate field or order parameter $\hat{\Phi}$ and we assume it uniform in space $\nabla \rho=0$. Minimize $\mathcal{E}$ with respect to $\rho$, and we obtain the particle density

$$
\rho_{0}=\frac{\mu}{\kappa v^{2}+g},
$$

which is suppressed by $v$ as compared with its value in the ground state (34). Plugging $\rho_{0}$ into the charge currents in Eq. (20), we find that the charge current $J$ vanishes $J_{i}=$ 0 . Nevertheless, the dipole currents $\mathcal{D}^{(a)}$ or the many-body current $\boldsymbol{\Xi}$ in Eq. (23) which after condensation reads

$$
\Xi_{i a}=-2 \rho_{0}^{2} K_{i a} \partial_{i} \partial_{a} \theta \quad \text { after condensation, }
$$

takes a finite value:

$$
\Xi_{s}=\kappa \rho_{0}^{2} \nu
$$

where $\Xi_{s}=\sqrt{\sum_{i, a=1}^{d}\left|\Xi_{i a}\right|^{2}}$. At $v=\sqrt{\frac{g}{3 \kappa}}, \Xi_{s}$ reaches its $\operatorname{maximum}\left(\Xi_{s}\right)_{\max }$,

$$
\left(\Xi_{s}\right)_{\max }=\frac{3 \sqrt{3 \kappa} \mu^{2}}{16 \sqrt{g^{3}}} .
$$

We conclude that $\left(\Xi_{S}\right)_{\max }$ plays the similar role as the critical charge current of a conventional superfluid phase. It means that a fractonic superfluid phase can survive when no induced charge currents appear and $\Xi_{s}$ is smaller than $\left(\Xi_{s}\right)_{\max }$ in Eq. (44) and that the many-body currents can flow dissipationlessly. For the diagonal case $K_{i j}=\frac{1}{2} \kappa \delta_{i j}$ under a proper boundary condition, we can obtain the same results as the isotropic case. Therefore, the many-body current $\boldsymbol{\Xi}$ is the supercurrent of the fractonic superfluid phase.

\section{Topological vortices}

A conventional $U(1)$ superfluid has vortex excitations and their topological nature can be characterized by vorticity as a close line integral over charge currents that are proportional to $\nabla \theta$. It is a different story for a fractonic superfluid phase due to its complicated form in Eq. (1). Instead, a many-body current $\boldsymbol{\Xi}$ in Eq. (23) that arises from purely two-particle correlated hopping directly gives vortex's topological number. 
Given a ground-state wave function in Eq. (34) for a fractonic phase, we are ready to discuss its topological nature. Our guiding rule is to relate vortex degree of freedom as the singular component of field $\theta$ to a winding number. The velocities in Eqs. (29) and (30) reduce to simpler forms after condensation with a uniform density distribution $\rho=\rho_{0}$,

$$
\begin{gathered}
v_{i}=-2 \rho_{0} \sum_{j}^{d} K_{i j} \partial_{i} \partial_{j}^{2} \theta \quad \text { after condensation, } \\
v_{i}^{(a)}=x^{a} v_{i}+2 K_{i a} \rho_{0} \partial_{i} \partial_{a} \theta \quad \text { after condensation. }
\end{gathered}
$$

Topological number in a conventional superfluid is embedded in current vorticity. A vorticity can be expressed as a winding number around the vortex core. Nevertheless, for general $K_{i j}$, vorticities of $\mathbf{v}$ and $\mathbf{v}^{(a)}$ are no longer topologically invariant. In other words, $\oint_{C} \mathbf{v} \cdot d \mathbf{r}$ and $\oint_{C} \mathbf{v}^{(a)} \cdot d \mathbf{r}$ depend on local geometry of a closed path $C$ since $\mathbf{v}_{i}$ and $\mathbf{v}_{i}^{(a)}$ are not closed if using the terminology of differential forms.

As stated in Sec. II B, the current $\boldsymbol{\Xi}$ arises from a purely many-body effect. By contracting one of the two indexes of $\boldsymbol{\Xi}$ in Eq. (42) after condensation, we can construct a new vector field $\mathbf{U}$

$$
U_{i}=\sum_{a=1}^{d} K_{i a}^{-1} x^{a} \Xi_{i a}=-\sum_{a=1}^{d} 2 \rho_{0}^{2} x^{a} \partial_{i} \partial_{a} \theta .
$$

The prefactor $K_{i a}^{-1}$ is introduced to absorb anisotropy in $K_{i j}$ and $x^{a}$ is to decrease degree of derivative. If some $K_{i j}$ vanish, then the definition of $\mathbf{\Xi}$ should be understood by taking the limit $K_{i j} \rightarrow 0$. Thus, Eq. (47) works for general $K_{i j}$. The vorticity $\ell$ associated with $\mathbf{U}$ can be calculated directly,

$$
\begin{aligned}
\ell & =\oint_{C} \mathbf{U} \cdot d \mathbf{r} \\
& =-\sum_{a=1}^{d} \oint_{C}\left[\nabla\left(x^{a} 2 \rho_{0}^{2} \partial_{a} \theta\right)-\left(\nabla x^{a}\right) 2 \rho_{0}^{2} \partial_{a} \theta\right] \cdot d \mathbf{r} \\
& =2 \rho_{0}^{2} \oint_{C} \nabla \theta \cdot d \mathbf{r}=2 \rho_{0}^{2} 2 \pi N,
\end{aligned}
$$

where $C$ is a closed loop in $d$ spatial dimensions and $N$ is a summation over winding numbers of vortices surrounded by the loop $C$. Therefore, $\ell$ can be utilized to characterize the topological nature of vortices. The vanishing of the first term in the second line of Eq. (48) is due to smoothness of $\partial_{a} \theta$ although $\theta$ is a multivalued function, since the microscopic model in Eq. (1) appears in a flat-band platform. From the construction of $\Xi$ in Eq. (23), we can conclude that topological properties arise from a purely many-body effect.

Pictorially, Figs. 1(a) and 1(b) show the configurations of $\mathbf{U}$ of a single vortex and a pair of vortex and antivortex, respectively, for simplicity, in two spatial dimensions. In Fig. 1(a) $\mathbf{U}$ circulates around the vortex core marked by a black point. Since we are only concerned about the topological nature, we choose $\theta$ field of a single vortex as $\theta=\arctan x^{2} / x^{1}$ that belongs to the same topological sector with exact vortex solutions to Gross-Pitaevskii equation in Eq. (24). The configurations of different vector fields in Eqs. (45), (46), and (47) are plotted in Fig. 2. Obviously,

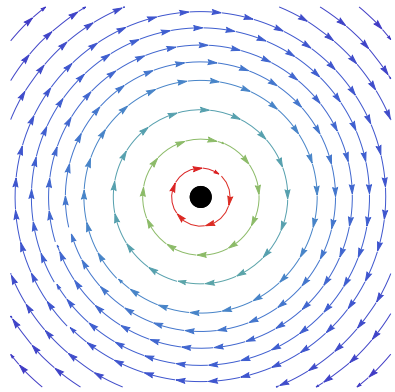

(a)

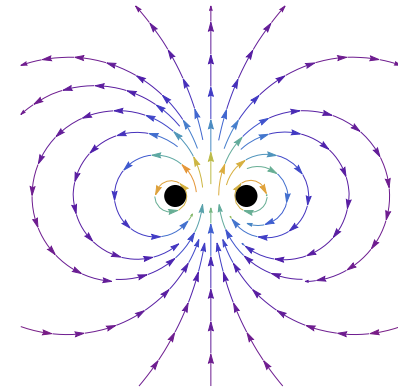

(b)
FIG. 1. Illustration of vector field $\mathbf{U}$ in Eq. (47) for (a) a single vortex $\theta=\arctan \frac{x^{2}}{x^{1}}$ and (b) a pair of vortex and its antivortex, for simplicity, in two spatial dimensions. The color and direction of arrow denote the strength (red $>$ blue) and direction of $\mathbf{U}$.

charge currents no longer wind around the vortex core, which is different from a conventional superfluid phase. Different choices of $K_{i j}$ induce different charge current configurations in Figs. 2(d), 2(g) and 2(j). Especially, when $\theta$ configuration appears as an exact solution to Gross-Pitaevskii equation in Eq. (24) for isotropic $K_{i j}$ in Fig. 2(a), charge currents vanish, which sharpens the failure of extracting topological number from charge currents. In Fig. 3, the configurations of different vector fields in Eqs. (45), (46), and (47) of a pair of vortex and antivortex [Fig. 1(b)] are depicted. The current distributions in Fig. 3 will be helpful in the analysis of interaction between vortices, which is left to future work.

In conclusion, we identify a fractonic superfluid phase. Different from a conventional superfluid phase, the topological vortices are characterized by the many-body current $\mathbf{\Xi}$ from the pure effect of two particles' correlated hopping and the corresponding supercurrents turn out to be the many-body currents $\boldsymbol{\Xi}$. Although for simplicity only vortex configurations in two spatial dimensions is discussed, a vortex in three spatial dimensions that has a line as its core has a similar structure. In Sec. IV, we will consider quantum fluctuations against stability of a fractonic superfluid phase. Although quantum fluctuation can destroy superfluidity in a purely two dimensional system, a fractonic superfluid phase may still stay stable in a quasi two spatial dimensions by the interlayer coupling.

\section{QUANTUM FLUCTUATIONS}

Quantum fluctuations can cause instability of a superfluid phase (i.e., ODLRO established in classical analysis). In this section we target on this problem. We first derive an effective theory for phase fluctuations based on our microscopic manyfracton model in Eq. (1) and then deal with stability of a fractonic superfluid phase.

\section{A. Effective theory of the microscopic model: Coherence length and effective Lagrangian}

To include quantum fluctuations, without loss of generality, we start with one classical field configuration $\phi_{0}(\mathbf{x}, t)=$ $\sqrt{\rho_{0}}$. Around it, $\phi(\mathbf{x}, t)=\sqrt{\rho_{0}+\rho(\mathbf{x}, t)} e^{i \theta(\mathbf{x}, t)}$ where two real fields $\rho(\mathbf{x}, t)$ and $\theta(\mathbf{x}, t)$ represent density and phase 


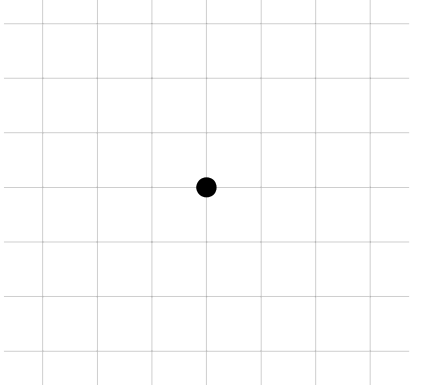

(a)

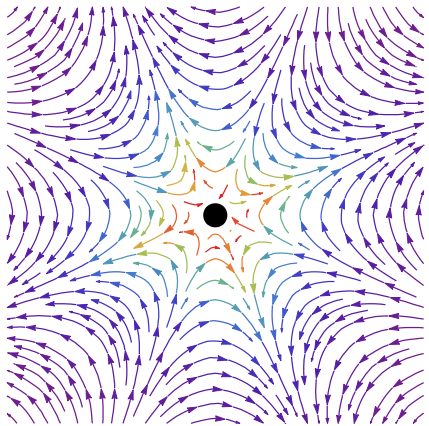

(d)

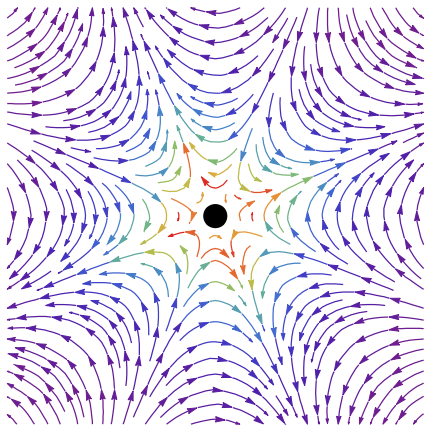

(g)

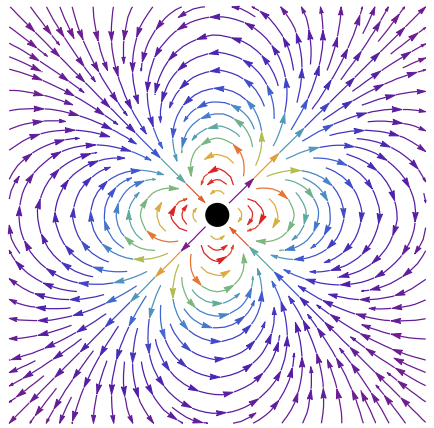

(j)

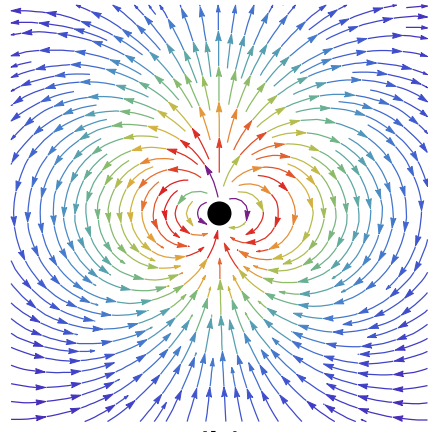

(b)

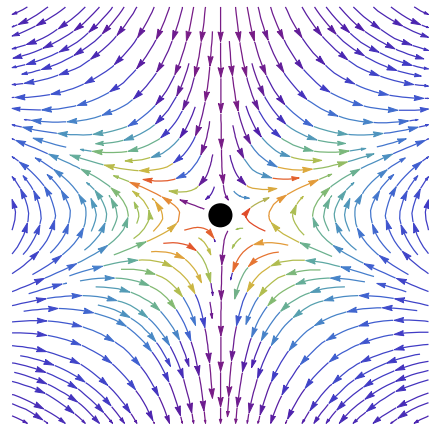

(e)

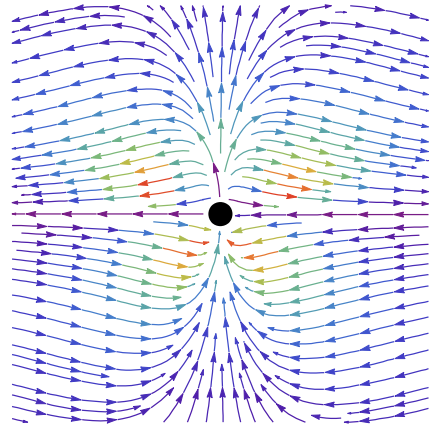

(h)

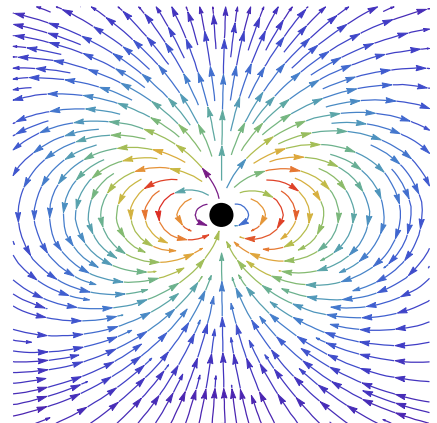

(k)

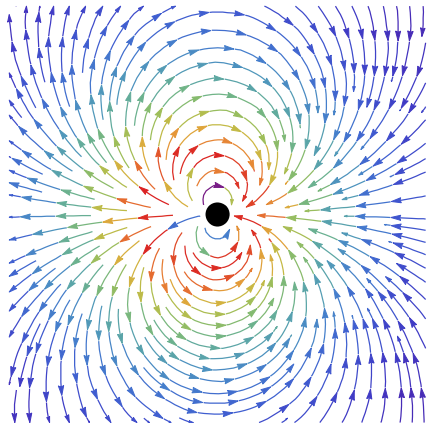

(c)

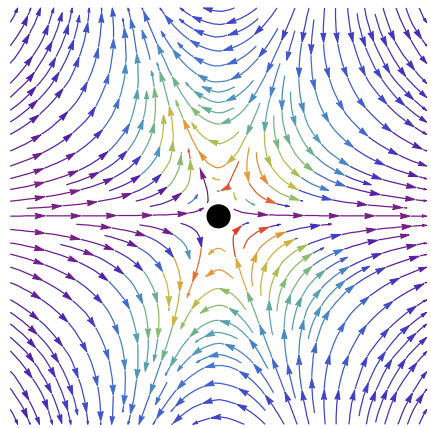

(f)

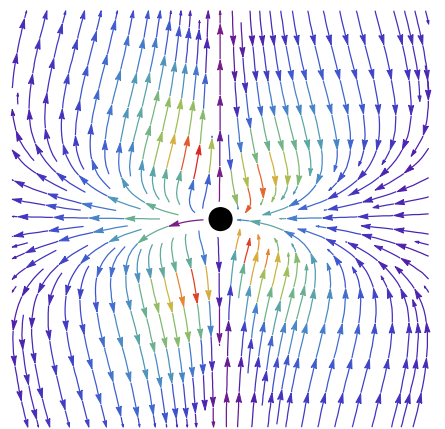

(i)

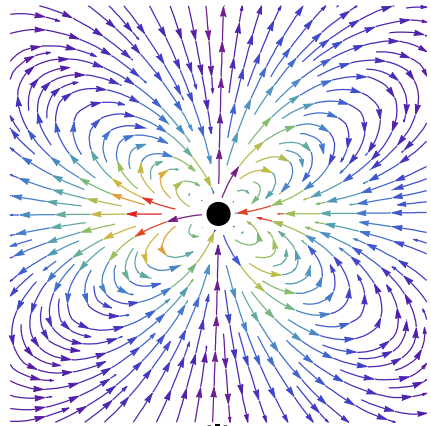

(l)

FIG. 2. Illustration of hydrodynamic fields in fractonic superfluid in the presence of a single vortex, for simplicity, in two spatial dimensions. The direction of an arrow denotes direction of fields and color characterizes strength. The vortex core in a vortex configuration of $\boldsymbol{\Xi}$ in Eq. (47) is marked by a black dot. The three columns from left to right depict velocity fields $\mathbf{v}$ in Eq. (45), $\mathbf{v}^{(1)}$ and $\mathbf{v}^{(2)}$ in Eq. (46), respectively. The four rows from top to bottom correspond to different coupling constants: isotropic $K_{i j}=\frac{1}{2} \kappa$, intermediate $K_{i j}=\left(\begin{array}{c}0.5 \kappa \\ 0.25 \kappa\end{array} \begin{array}{c}0.25 \kappa \\ 0.5 \kappa\end{array}\right)$, diagonal $K_{i j}=\frac{1}{2} \kappa \delta_{i j}$, and $K_{i j}=\left(\begin{array}{cc}1.5 \kappa & \kappa \\ \kappa & 0.5 \kappa\end{array}\right)$ with a positive constant $\kappa$. The vector fields $\Xi$ in these cases share the same configuration as in Fig. 1(b). The charge current for isotropic $K_{i j}$ vanishes in (a) where $\theta$ is an exact solution to Gross-Pitaevskii equation in Eq. (24). The vorticities of charge and dipole velocity fields are not topological. All the velocity fields are obtained under the same field $\theta=\arctan \frac{x^{2}}{x^{1}}$ with winding number $N=1$. 


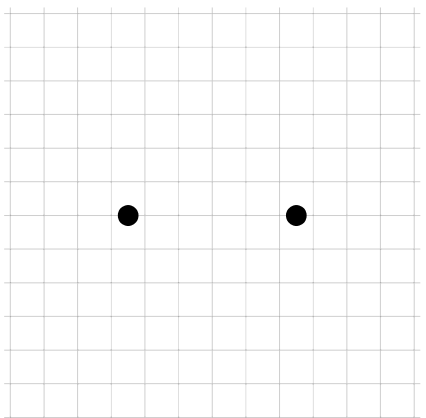

(a)

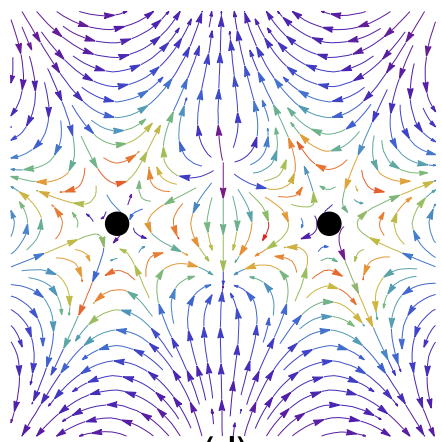

(d)

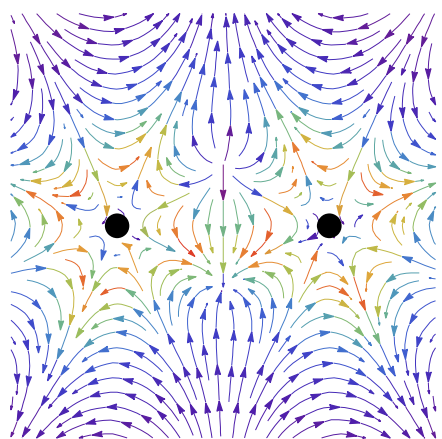

(g)

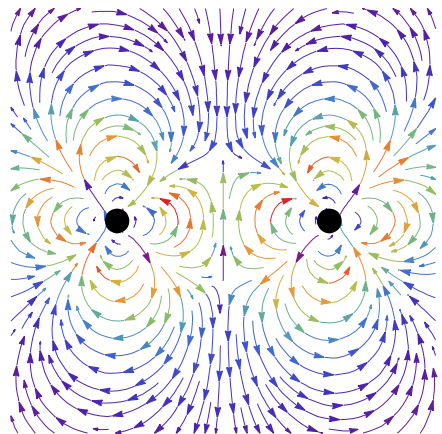

(j)

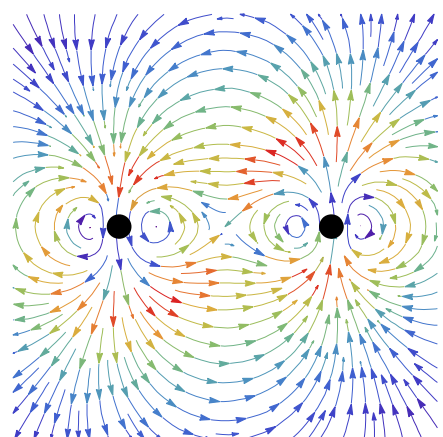

(b)

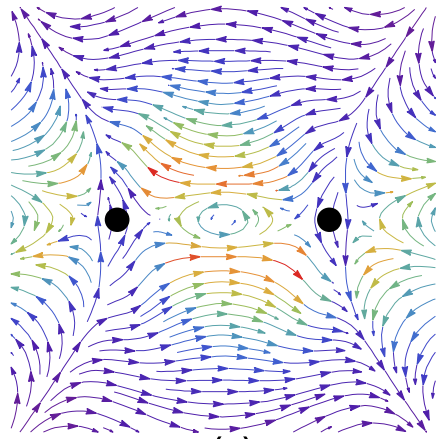

(e)

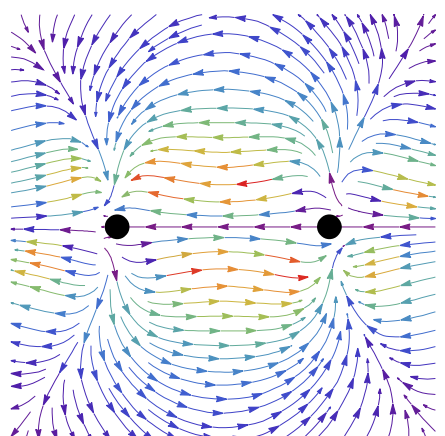

(h)

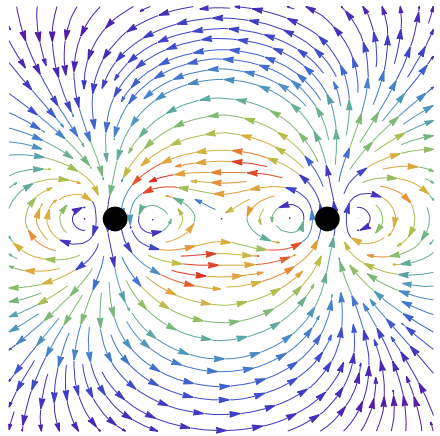

(k)

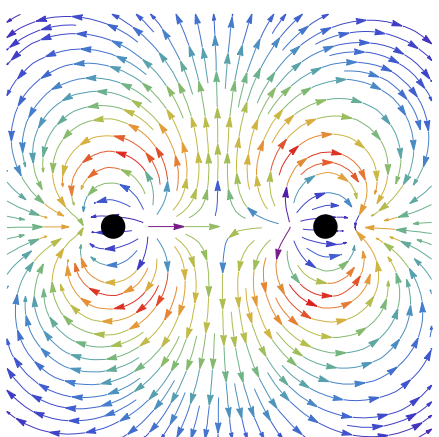

(c)

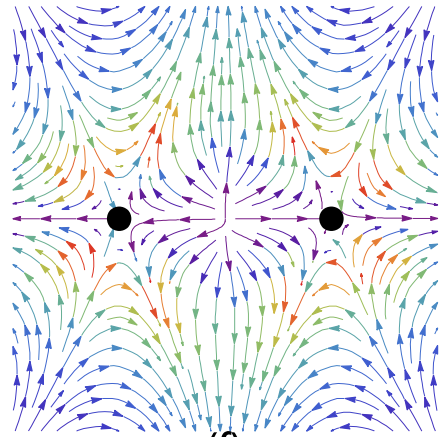

(f)

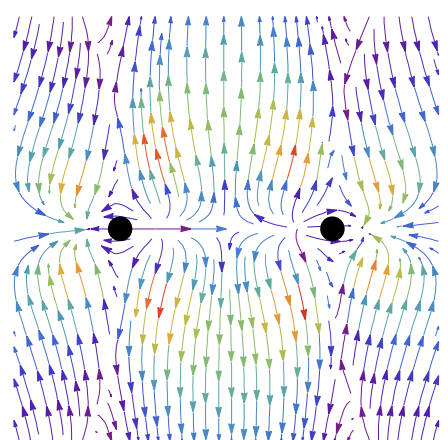

(i)

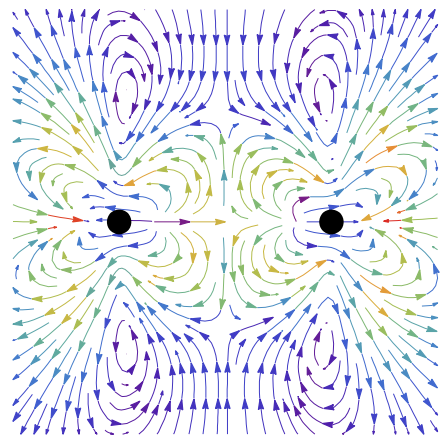

(I)

FIG. 3. Illustration of hydrodynamic fields in fractonic superfluid in the presence of a pair of vortex-antivortex, for simplicity, in two spatial dimensions. The direction of an arrow denotes direction of fields and color characterizes strength. The vortex core in a vortex configuration of $\boldsymbol{\Xi}$ in Eq. (47) is marked by a black dot. The three columns from left to right depict velocity fields $\mathbf{v}$ in Eq. (45), $\mathbf{v}^{(1)}$ and $\mathbf{v}^{(2)}$ in Eq. (46), respectively. The four rows from top to bottom correspond to different coupling constants as those in Fig. 2. The two vortices have the winding number 1 (left) and -1 (right). 
fluctuations respectively. We have removed the Greek letter $\delta$ in $\delta \rho$ for notation simplicity. We remark that the field $\theta$ is angular valued and should be defined $\bmod 2 \pi$. To the second order, we can derive the effective Lagrangian corresponding to the microscopic model in Eq. (1),

$$
\begin{aligned}
\mathcal{L}= & -\rho \partial_{t} \theta-\sum_{i, j}^{d} K_{i j}\left[\rho_{0}^{2}\left(\partial_{i} \partial_{j} \theta\right)^{2}+\frac{1}{4}\left(\partial_{i} \partial_{j} \rho\right)^{2}\right] \\
& -\sum_{i}^{d} G_{i}\left(\partial_{i} \rho\right)^{2}-\frac{g}{2} \rho^{2} .
\end{aligned}
$$

The density fluctuation field $\rho$ should satisfy a condition as an auxiliary field $\frac{\delta \mathcal{L}}{\delta \rho}=0$. The solution takes the form in the momentum space as

$$
\rho(\omega, \mathbf{k})=\frac{-i \omega \theta(\omega, \mathbf{k})}{g+\frac{1}{2}\left(\sum_{i, j}^{d} K_{i j} k_{i}^{2} k_{j}^{2}\right)+2\left(\sum_{i}^{d} G_{i} k_{i}^{2}\right)} .
$$

In the long-wave length limit,

$$
g \gg \frac{1}{2}\left(\sum_{i, j}^{d} K_{i j} k_{i}^{2} k_{j}^{2}\right)+2\left(\sum_{i}^{d} G_{i} k_{i}^{2}\right),
$$

which requires an upper bound for momentum $|\mathbf{k}| \ll 2 \pi \xi_{\text {coh }}^{-1}$. $\xi_{\text {coh }}$ is the coherence length of the fractonic superfluid and is determined when the right-hand side and left-hand side in Eq. (51) equal. So we can safely make the approximation: $\rho(\omega, \mathbf{k})=-\frac{i \omega \theta(\omega, \mathbf{k})}{g}$. Therefore, we obtain an effective Lagrangian for field $\theta$,

$$
\mathcal{L}=\frac{1}{2 g}\left(\partial_{t} \theta\right)^{2}-\rho_{0}^{2} \sum_{i, j}^{d} K_{i j}\left(\partial_{i} \partial_{j} \theta\right)^{2} .
$$

In Eq. (52) since the $G_{i}$ term is less relevant, we have neglected it. The same dispersion relation for the field $\theta$ can be given through a classical analysis and we will not go into details. There are two issues to be addressed here. First, the effective theory in Eq. (52) is valid only when the length scale is much larger than the coherence length $\xi_{\text {coh }}$. Thus, the prediction power of the effective theory becomes questionable for length scale smaller than or comparable to $\xi_{\text {coh. Second, }}$ $\theta$ is in fact an angular-valued field and is defined $\bmod 2 \pi$. Now we just treat as a real valued field, which is enough for description of the fixed point physics of the fractonic superfluid phase.

Although the broken symmetry $\mathcal{G}$ has $d+1$ generators, we only have one gapless mode $\theta$ in Eq. (52). Notice that any vacuum with a broken $U(1)$ charge symmetry also is one for symmetry generated by $Q^{(a)}$ and the main effect of a charge $Q^{(a)}$ is to impose a strong constraint on current $J_{i}$ so that the Goldstone mode has a higher-order dispersion relation.

\section{B. Stability of ODLRO: Correlation functions and specific heat}

We are ready to include the effect of quantum fluctuations on correlation $C(\mathbf{x})$ in Eq. (37). The correlator $C(\mathbf{x})$ is related to an equal-time Green function of the Golstone mode $\theta(\mathbf{x}, 0)$,

$$
C(\mathbf{x})=\rho_{0} e^{-\frac{1}{2}\left\langle[\theta(\mathbf{x}, 0)-\theta(0,0)]^{2}\right\rangle},
$$

TABLE II. Comparison between correlators in Eq. (53) of conventional superfluid and isotropic fractonic superfluid at zero temperature after quantum fluctuations are included. $d$ is spatial dimension. The effective theories are given by Eqs. (59) and (57), respectively. The former has $c=\sqrt{\kappa g \rho_{0}}$ and coherence length $\xi_{\text {coh }}=$ $2 \pi \sqrt{\kappa /\left(4 \rho_{0} g\right)}$ while the latter with isotropic coupling constant $K_{i j}=$

\begin{tabular}{|c|c|c|c|c|}
\hline \multirow{2}{*}{$\frac{d}{1}$} & \multicolumn{2}{|c|}{ Conventional system } & \multicolumn{2}{|c|}{ Many-fracton system } \\
\hline & $\rho_{0} e^{-\frac{\gamma g}{4 \pi c}}\left(\pi r / \xi_{\mathrm{coh}}\right)^{-\frac{g}{2 \pi c}}$ & $\mathrm{AO}$ & $\rho_{0} e^{-\frac{g}{2 c}\left(\pi r-\xi_{\operatorname{coh}} / \pi^{\frac{3}{2}}\right)}$ & $x$ \\
\hline 2 & $\rho_{0} e^{-\frac{g}{2 \pi c} \xi_{\mathrm{coh}}^{-1}}$ & $\checkmark$ & $\rho_{0} e^{-\frac{\gamma g}{4 \pi c}}\left(r / \xi_{\mathrm{coh}}\right)^{-\frac{g}{2 \pi c}}$ & $\mathrm{AO}$ \\
\hline$\geqslant 3$ & $\rho_{0} e^{-g \frac{\pi^{\frac{d-3}{2}}}{2(d-1) c} \xi_{\mathrm{coh}}^{1-d}}$ & $\checkmark$ & $\rho_{0} e^{-\frac{g}{c} \frac{\frac{d}{2}-2}{2(d-2)} \xi_{\mathrm{coh}}^{2-d}}$ & $\checkmark$ \\
\hline
\end{tabular}
$\frac{1}{2} \kappa$ has $c=\sqrt{\kappa g \rho_{0}^{2}}$ and coherence length $\xi_{\text {coh }}=2 \pi \sqrt[4]{\kappa / 4 g}$. A manyfracton system is fully disordered (marked by $\times$ ) in $d=1$ and algebraically ordered (AO) in $d=2$. It has a stable ODLRO, i.e., a true superfluid (marked by $\checkmark$ ), when $d \geqslant 3$ at zero temperature. Here $\gamma$ is the Euler's constant.

where the equal-time Green function can be calculated in the momentum space:

$$
\begin{aligned}
& \left\langle[\theta(\mathbf{x}, 0)-\theta(0,0)]^{2}\right\rangle \\
& \quad=\int \frac{d^{d} \mathbf{k} d \omega}{(2 \pi)^{d+1}}\left(2-2 e^{-i \mathbf{k} \cdot \mathbf{x}}\right)\langle\theta(\omega, \mathbf{k}) \theta(-\omega,-\mathbf{k})\rangle .
\end{aligned}
$$

Equation (54) is hard to deal with exactly for a general $K_{i j}$. In the following, we consider two specified cases.

\section{Isotropic case $K_{i j}=\frac{1}{2} \kappa$ for any $i, j$}

We introduce a factor $\frac{1}{2}$ in $K_{i j}$ to simplify our expressions. In this case, the Goldstone mode $\theta$ has a quadratic dispersion relation,

$$
\omega=\sqrt{\kappa g \rho_{0}^{2}}|\mathbf{k}|^{2} \equiv c|\mathbf{k}|^{2},
$$

and it recovers a rotational symmetry. The coherence length $\xi_{\text {coh }}$ is determined by equation $g=\frac{1}{4} \kappa\left(\frac{2 \pi}{\xi_{\mathrm{coh}}}\right)^{4}$, where the $G_{i}$ term is less relevant for the upper bound of momentum. So we have

$$
\xi_{\mathrm{coh}}=2 \pi\left(\frac{\kappa}{4 g}\right)^{\frac{1}{4}}
$$

The Lagrangian

$$
\mathcal{L}=\frac{1}{2 g}\left(\partial_{t} \theta\right)^{2}-\frac{1}{2 g} c^{2}\left(\nabla^{2} \theta\right)^{2}
$$

possesses a Lifshitz spacetime symmetry and is related to nonrelativistic gravity [65-67].

The asymptotic behavior of $C(\mathbf{x})$ has been listed in Table II. We can find that only when our space dimension $d>2$ does a superfluid survive quantum fluctuations. The correlator $C(\mathbf{x})$ approaches zero in dimension $d=1$ and 2 in large distance. We point out that $C(\mathbf{x})$ decays in a power-law pattern in $d=2$, which is similar to a conventional superfluid in $d=1$. Another aspect of the higher-order dispersion in Eq. (55) is 
specific heat capacity:

$$
c_{v}=(T / c)^{\frac{d}{2}} \frac{\Omega_{d-1}}{2(2 \pi)^{d}} \Gamma\left(\frac{d}{2}+2\right) \zeta\left(\frac{d+2}{2}\right),
$$

where $\Omega_{d-1}$ is surface area of unit $(d-1)$ sphere and $\zeta(s)$ is the Riemann $\zeta$ function. From Eq. (58), $c_{v}$ is proportional to $T^{\frac{d}{2}}$ in $d$ space dimensions. When a spatial dimension is lower than 3 , the specific heat capacity is physically meaningless. The result in Eq. (58) for $d=3$ is valid under our assumption of existence of Goldstone mode at finite temperature. On the other hand, for a conventional superfluid with Lagrangian $\mathcal{L}=i \phi^{*} \partial_{t} \phi-\frac{1}{2} \kappa|\nabla \phi|^{2}-V(\phi)$ with $V(\phi)$ in Eq. (12), the effective theory for the Goldstone mode is

$$
\mathcal{L}=\frac{1}{2 g}\left(\partial_{t} \theta\right)^{2}-\frac{1}{2 g} c^{2}(\nabla \theta)^{2},
$$

where Goldstone mode has a linear dispersion relation $\omega=$ $\sqrt{k g \rho_{0}}|\mathbf{k}| \equiv c|\mathbf{k}|$ and quantum fluctuation will kill a superfluid phase in one spatial dimension at zero temperature. Table II makes a comparison between a conventional and fractonic superfluid phase. Interestingly, Ref. [61] discussed a similar effective Lagrangian from a different higher-derivative model where excitons [71] form a condensate. In our context, the effective Lagrangian in Eq. (57) is a description of fractonic superfluids in the isotropic case, whose microscopic origin is given by Eq. (1) and the order parameter is given by $\langle\hat{\Phi}\rangle$ in Table I.

\section{Diagonal case $K_{i j}=\frac{1}{2} \kappa \delta_{i j}$}

Now the Goldstone mode $\theta$ has a dispersion spectrum

$$
\omega=\sqrt{\kappa g \rho_{0}^{2}} \sqrt{\sum_{i}^{d} k_{i}^{4}} \equiv c \sqrt{\sum_{i}^{d} k_{i}^{4}} .
$$

It does not have a rotational symmetry. We can arrive at the same conclusion as the isotropic case.

The above analysis just demonstrates that a superfluid phase or ODLRO cannot survive against quantum fluctuation when the spatial dimension is lower than three at zero temperature. In particular, in $d=2$ quantum fluctuations only allow an algebraic order. However, similar to superconductivity in 2D materials, interlayer couplings or a quasi-2D system can stabilize the fractonic superfluid against quantum fluctuations. Thermal effect may destroy a fractonic superfluid phase and the related results will be present in future work. In Appendix A, we aim to discuss general many-fracton models.

\section{CONCLUSION}

In this paper, we have studied a many-fracton model in the microscopic Hamiltonian (1) that lacks of mobility of a single particle. The model in Eq. (1) conserves both charge and total dipole moments. We have derived nontrivial EulerLagrange equation and the Noether currents. By taking a Mexican-hat potential, we deduce a time-dependent GrossPitaevskii-type equations. We finally end up with fractonic superfluidity from both classical and quantum levels of length scale much larger than coherence length $\xi_{\text {coh }}$, including supercurrent, topological vortices, ODLRO stability against gapless Goldstone modes and specific heat in low temperatures. The Hamiltonian in Eq. (1) is expected to be realized in cold atomic gas subjected to an optical lattice [68,69], especially when a trap is considered, and opens a new horizon to search for exotic phases of matter. There are many interesting directions for future investigation. For example, we can discuss a fractonic superconducting phase by allowing a fracton field $\hat{\Phi}$ in Eq. (1) to satisfy anticommutation relations with possible pairing field $\Delta_{i j} \sim \hat{\Phi} \partial_{i} \partial_{j} \hat{\Phi}-\partial_{i} \hat{\Phi} \partial_{j} \hat{\Phi}$. And then, the BECBCS crossover of a fracton system may show interesting physical consequence. Despite of highly nonlinearity, numerically solving the Gross-Pitaevskii equations will be very attractive. One can consider fractonic versions of other types of ordered phases, such as nematic and stripe orders and discuss their competitions. One may also consider a symmetric phase formed by condensing unconventional vortices in the fractonic superfluid. By decorating on-site symmetry charge on vortices, one may construct SPTs with both higher rank symmetry and usual on-site symmetry, following the similar methods in usual SPT constructions [12-15,17].

\section{ACKNOWLEDGMENTS}

We thank Meng Cheng and Juven Wang for useful discussions. The work was supported by the SYSU startup grant and NSFC Grant No. 11847608.

\section{APPENDIX: GENERAL MANY-FRACTON MODELS}

We can generalize the many-fracton model in Eq. (1) into a large class. We begin with a Hamiltonian $H=\int d^{d} \mathbf{x} \mathcal{H}_{N}$ where the Hamiltonian density $\mathcal{H}_{N}$ reads

$$
\begin{aligned}
\mathcal{H}_{N}= & \sum_{i_{1} i_{2} \cdots i_{N+1}}^{d} K_{i_{1} i_{2} \cdots i_{N+1}}\left(\hat{\Phi}^{\dagger}\right)^{N+1}\left(\nabla_{i_{1} i_{2} \cdots i_{N+1}} \log \hat{\Phi}^{\dagger}\right) \hat{\Phi}^{N+1} \\
& \times\left(\nabla_{i_{1} i_{2} \cdots i_{N+1}} \log \hat{\Phi}\right)+V\left(\hat{\Phi}^{\dagger}, \hat{\Phi}\right),
\end{aligned}
$$

where $\nabla_{i_{1} i_{2} \cdots i_{n}}=\partial_{i_{1}} \partial_{i_{2}} \cdots \partial_{i_{n}}$ and the summation for each index is over all spatial dimensions. The coupling constant $K_{i_{1} i_{2} \cdots i_{N+1}}$ can be anisotropic and it is fully symmetric with its indexes. When $N=0$, Eq. (A1) reduces to a Gaussian theory and when $N=1$, it reduces to the many-fracton model in Eq. (1) except $G_{i}$ term. Here some models are listed

$$
\begin{gathered}
\mathcal{H}_{0}=\sum_{i}^{d} K_{i} \partial_{i} \hat{\Phi}^{\dagger} \partial_{i} \hat{\Phi}+V\left(\hat{\Phi}^{\dagger}, \hat{\Phi}\right), \\
\mathcal{H}_{2}=\sum_{i, j, k}^{d} K_{i j k}\left[2 \partial_{i} \hat{\Phi}^{\dagger} \partial_{j} \hat{\Phi}^{\dagger} \partial_{k} \hat{\Phi}^{\dagger}-3 \hat{\Phi}^{\dagger} \partial_{i} \hat{\Phi}^{\dagger} \partial_{j} \partial_{k} \hat{\Phi}^{\dagger}+\left(\hat{\Phi}^{\dagger}\right)^{2} \partial_{i} \partial_{j} \partial_{k} \hat{\Phi}^{\dagger}\right] \cdot\left[2 \partial_{i} \hat{\Phi} \partial_{j} \hat{\Phi} \partial_{k} \hat{\Phi}-3 \hat{\Phi} \partial_{i} \hat{\Phi} \partial_{j} \partial_{k} \hat{\Phi}+\hat{\Phi}^{2} \partial_{i} \partial_{j} \partial_{k} \hat{\Phi}\right]+V\left(\hat{\Phi}^{\dagger}, \hat{\Phi}\right),
\end{gathered}
$$




$$
\begin{aligned}
\mathcal{H}_{3}= & \sum_{i, j, k, l}^{d} K_{i j k l}\left[6 \partial_{i} \hat{\Phi}^{\dagger} \partial_{j} \hat{\Phi}^{\dagger} \partial_{k} \hat{\Phi}^{\dagger} \partial_{l} \hat{\Phi}^{\dagger}-12 \hat{\Phi}^{\dagger} \partial_{i} \hat{\Phi}^{\dagger} \partial_{j} \hat{\Phi}^{\dagger} \partial_{k} \partial_{l} \hat{\Phi}^{\dagger}+4\left(\hat{\Phi}^{\dagger}\right)^{2} \partial_{i} \hat{\Phi}^{\dagger} \partial_{j} \partial_{k} \partial_{l} \hat{\Phi}^{\dagger}\right. \\
& \left.+\left(\hat{\Phi}^{\dagger}\right)^{2}\left(3 \partial_{i} \partial_{j} \hat{\Phi}^{\dagger} \partial_{k} \partial_{l} \hat{\Phi}^{\dagger}-\hat{\Phi}^{\dagger} \partial_{i} \partial_{j} \partial_{k} \partial_{l} \hat{\Phi}^{\dagger}\right)\right]\left[6 \partial_{i} \hat{\Phi} \partial_{j} \hat{\Phi} \partial_{k} \hat{\Phi} \partial_{l} \hat{\Phi}-12 \hat{\Phi} \partial_{i} \hat{\Phi} \partial_{j} \hat{\Phi} \partial_{k} \partial_{l} \hat{\Phi}\right. \\
& \left.+4 \hat{\Phi}^{2} \partial_{i} \hat{\Phi} \partial_{j} \partial_{k} \partial_{l} \hat{\Phi}+\hat{\Phi}^{2}\left(3 \partial_{i} \partial_{j} \hat{\Phi} \partial_{k} \partial_{l} \hat{\Phi}-\hat{\Phi} \partial_{i} \partial_{j} \partial_{k} \partial_{l} \hat{\Phi}\right)\right]+V\left(\hat{\Phi}^{\dagger}, \hat{\Phi}\right) .
\end{aligned}
$$

Under the standard coherent-state path integral, we can write down the Lagrangian $\mathcal{L}=i \phi^{*} \partial_{t} \phi-\mathcal{H}\left(\phi^{*}, \phi\right)$. Although $\log \phi$ is a multivalued function and has singularity, the kinetic term turns out to be well defined. The system in Eq. (A1) is invariant under a transformation

$$
\phi \rightarrow \exp (i \delta \theta) \phi
$$

where $\delta \theta$ is polynomials of degree $N$ of local coordinates

$$
\delta \theta=\sum_{i_{1} \cdots i_{N}} \mathcal{D}_{i_{1} i_{2} \cdots i_{N}} x^{i_{1}} x^{i_{2}} \cdots x^{i_{N}}+\cdots+\sum_{i} \mathcal{D}_{i} x^{i}+\mathcal{D},
$$

where $\mathcal{D}_{i_{1} \ldots i_{l}}$ is a symmetric real tensor of rank- $l$ with respect to spatial indexes. And the related conserved charges have the form as

$$
Q^{\left[C\left(x^{a}\right)\right]}=\int d^{d} \mathbf{x} \rho C\left(x^{a}\right),
$$

where $C\left(x^{a}\right)$ is as a homogeneous polynomials with degree$p$ and $p \leqslant N$. We dub a symmetry generated by charges in
Eq. (A7) as a rank-Nsymmetry [51,70]. In this sense, a global $U(1)$ is a rank-0 symmetry and $\mathcal{G}$ for Hamiltonian in Eq. (1) is a rank-1 symmetry.

Now we focus on an isotropic coupling constant $\mathcal{D}_{i_{1} i_{2} \cdots i_{N+1}}=\frac{1}{2} \kappa$. If we take a Mexican-hat potential chemical potential $\mu>0$, then we have degenerate vacuum with finite uniform density distribution $\rho=\rho_{0}$. Through the same processes, we can derive an effective theory for the quantum fluctuation field $\theta$ after condensation,

$$
\mathcal{L}=\frac{1}{2 g}\left(\partial_{t} \theta\right)^{2}-\frac{1}{2 g} c^{2}\left(\nabla^{N+1} \theta\right)^{2} .
$$

The effective theory describes Goldstone mode $\theta$ with a dispersion relation $\omega=\sqrt{\kappa g \rho_{0}^{N+1}}|\mathbf{k}|^{N+1} \equiv c|\mathbf{k}|^{N+1}$. The calculation on the correlator $C(\mathbf{x})$ shows that $C(\mathbf{x})$ decays to zero when spatial dimension is lower than $d<N+2$ at zero temperature. In particular, $C(\mathbf{x})$ decays in a power-law pattern at zero temperature at spatial dimension $d=N+1$.
[1] C. J. Pethick and H. Smith, Bose-Einstein Condensation in Dilute Gases (Cambridge University Press, Cambridge, UK, 2008).

[2] P. M. Chaikin and T. C. Lubensky, Principles of Condensed Matter Physics, Vol. 1 (Cambridge University Press, Cambridge, UK, 2000).

[3] C. N. Yang, Concept of off-diagonal long-range order and the quantum phases of liquid he and of superconductors, Rev. Mod. Phys. 34, 694 (1962).

[4] A. J. Leggett, Bose-einstein condensation in the alkali gases: Some fundamental concepts, Rev. Mod. Phys. 73, 307 (2001).

[5] I. Bloch, J. Dalibard, and W. Zwerger, Many-body physics with ultracold gases, Rev. Mod. Phys. 80, 885 (2008).

[6] S. Giorgini, L. P. Pitaevskii, and S. Stringari, Theory of ultracold atomic fermi gases, Rev. Mod. Phys. 80, 1215 (2008).

[7] M. Lewenstein, A. Sanpera, V. Ahufinger, B. Damski, A. Sen, and U. Sen, Ultracold atomic gases in optical lattices: Mimicking condensed matter physics and beyond, Adv. Phys. 56, 243 (2007).

[8] L. D. Carr, D. DeMille, R. V. Krems, and J. Ye, Cold and ultracold molecules: Science, technology and applications, New J. Phys. 11, 055049 (2009).

[9] J. Dalibard, F. Gerbier, G. Juzeliūnas, and P. Öhberg, Colloquium: Artificial gauge potentials for neutral atoms, Rev. Mod. Phys. 83, 1523 (2011).

[10] I. M. Georgescu, S. Ashhab, and F. Nori, Quantum simulation, Rev. Mod. Phys. 86, 153 (2014).
[11] A. Celi, A. Sanpera, V. Ahufinger, and M. Lewenstein, Quantum optics and frontiers of physics: the third quantum revolution, Phys. Scr. 92, 013003 (2016).

[12] Z.-C. Gu, J. C. Wang, and X.-G. Wen, Multikink topological terms and charge-binding domain-wall condensation induced symmetry-protected topological states: Beyond chernsimons/bf field theories, Phys. Rev. B 93, 115136 (2016).

[13] P. Ye and Z.-C. Gu, Vortex-Line Condensation in Three Dimensions: A Physical Mechanism for Bosonic Topological Insulators, Phys. Rev. X 5, 021029 (2015).

[14] P. Ye and Z.-C. Gu, Topological quantum field theory of threedimensional bosonic abelian-symmetry-protected topological phases, Phys. Rev. B 93, 205157 (2016).

[15] AtMa P. O. Chan, P. Ye, and S. Ryu, Braiding with Borromean Rings in $(3+1)$-Dimensional Spacetime, Phys. Rev. Lett. 121, 061601 (2018).

[16] X. G. Wen and A. Zee, Compressibility and superfluidity in the fractional-statistics liquid, Phys. Rev. B 41, 240 (1990).

[17] X. Chen, Y.-M. Lu, and A. Vishwanath, Symmetry-protected topological phases from decorated domain walls, Nat. Commun. 5, 3507 (2014).

[18] C. Chamon, Quantum Glassiness in Strongly Correlated Clean Systems: An Example of Topological Overprotection, Phys. Rev. Lett. 94, 040402 (2005).

[19] S. Vijay, J. Haah, and L. Fu, A new kind of topological quantum order: A dimensional hierarchy of quasiparticles built from stationary excitations, Phys. Rev. B 92, 235136 (2015). 
[20] S. Vijay, J. Haah, and L. Fu, Fracton topological order, generalized lattice gauge theory, and duality, Phys. Rev. B 94, 235157 (2016).

[21] R. M. Nandkishore and M. Hermele, Fractons, Annu. Rev. Condens. Matter Phys. 10, 295 (2019).

[22] A. Prem, J. Haah, and R. Nandkishore, Glassy quantum dynamics in translation invariant fracton models, Phys. Rev. B 95, 155133 (2017).

[23] W. Shirley, K. Slagle, and X. Chen, Foliated fracton order from gauging subsystem symmetries, SciPost Phys. 6, 41 (2019).

[24] H. Ma, E. Lake, X. Chen, and M. Hermele, Fracton topological order via coupled layers, Phys. Rev. B 95, 245126 (2017).

[25] J. Haah, Local stabilizer codes in three dimensions without string logical operators, Phys. Rev. A 83, 042330 (2011).

[26] D. Bulmash and M. Barkeshli, Gauging fractons: immobile non-Abelian quasiparticles, fractals, and position-dependent degeneracies, Phys. Rev. B 100, 155146 (2019).

[27] A. Prem and D. J. Williamson, Gauging permutation symmetries as a route to non-Abelian fractons, SciPost Phys. 7, 068 (2019).

[28] D. Bulmash and M. Barkeshli, Generalized $U(1)$ gauge field theories and fractal dynamics, arXiv:1806.01855 [cond-mat.strel].

[29] K. T. Tian, E. Samperton, and Z. Wang, Haah codes on general three manifolds, Ann. Phys. 412, 168014 (2020).

[30] Y. You, D. Litinski, and F. von Oppen, Higher order topological superconductors as generators of quantum codes, Phys. Rev. B 100, 054513 (2019)

[31] H. Ma, M. Hermele, and X. Chen, Fracton topological order from the higgs and partial-confinement mechanisms of ranktwo gauge theory, Phys. Rev. B 98, 035111 (2018).

[32] K. Slagle and Y. B. Kim, Fracton topological order from nearest-neighbor two-spin interactions and dualities, Phys. Rev. B 96, 165106 (2017).

[33] G. B. Halász, T. H. Hsieh, and L. Balents, Fracton Topological Phases from Strongly Coupled Spin Chains, Phys. Rev. Lett. 119, 257202 (2017).

[34] K. T. Tian and Z. Wang, Generalized Haah codes and fracton models, arXiv:1902.04543 [quant-ph].

[35] W. Shirley, K. Slagle, and X. Chen, Fractional excitations in foliated fracton phases, Ann. Phys. 410, 167922 (2019).

[36] K. Slagle, D. Aasen, and D. Williamson, Foliated field theory and string-membrane-net condensation picture of fracton order, SciPost Phys. 6, 43 (2019).

[37] W. Shirley, K. Slagle, Z. Wang, and X. Chen, Fracton Models on Feneral Three-Dimensional Manifolds, Phys. Rev. X 8, 031051 (2018).

[38] A. Prem, S.-J. Huang, H. Song, and M. Hermele, Cage-Net Fracton Models, Phys. Rev. X 9, 021010 (2019).

[39] S. Pai, M. Pretko, and R. M. Nandkishore, Localization in Fractonic Random Circuits, Phys. Rev. X 9, 021003 (2019).

[40] S. Pai and M. Pretko, Dynamical Scar States in Driven Fracton Systems, Phys. Rev. Lett. 123, 136401 (2019).

[41] P. Sala, T. Rakovszky, R. Verresen, M. Knap, and F. Pollmann, Ergodicity-Breaking Arising from Hilbert Space Fragmentation in Dipole-Conserving Hamiltonians Phys. Rev. X 10, 011047 (2020).

[42] A. Kumar and A. C. Potter, Symmetry-enforced fractonicity and two-dimensional quantum crystal melting, Phys. Rev. B 100, 045119 (2019).

[43] M. Pretko, The fracton gauge principle, Phys. Rev. B 98, 115134 (2018).

[44] M. Pretko, Subdimensional particle structure of higher rank $u$ (1) spin liquids, Phys. Rev. B 95, 115139 (2017).

[45] M.-Y. Li and P. Ye, Exactly solvable fracton models for spatially extended excitations, arXiv:1909.02814 [cond-mat.str-el].

[46] M. Pretko, Generalized electromagnetism of subdimensional particles: A spin liquid story, Phys. Rev. B 96, 035119 (2017).

[47] L. Radzihovsky and M. Hermele, Fractons from Vector Gauge Theory, Phys. Rev. Lett. 124, 050402 (2020).

[48] A. Dua, I. H. Kim, M. Cheng, and D. J. Williamson, Sorting topological stabilizer models in three dimensions, Phys. Rev. B 100, 155137 (2019).

[49] A. Gromov, Chiral Topological Elasticity and Fracton Order, Phys. Rev. Lett. 122, 076403 (2019).

[50] J. Haah, Lattice quantum codes and exotic topological phases of matter, Ph.D. thesis, California Institute of Technology, 2013.

[51] A. Gromov, Towards Classification of Fracton Phases: The Multipole Algebra, Phys. Rev. X 9, 031035 (2019).

[52] Y. You, T. Devakul, S. L. Sondhi, and F. J. Burnell, Fractonic Chern-Simons and BF theories arXiv:1904.11530 [condmat.str-el].

[53] J. Sous and M. Pretko, Fractons from polarons and hole-doped antiferromagnets: Microscopic realizations, arXiv:1904.08424 [cond-mat.str-el].

[54] J. Wang and K. Xu, Higher-rank tensor field theory of nonAbelian fracton and embeddon, arXiv:1909.13879 [hep-th].

[55] S. Pai and M. Pretko, Fractonic line excitations: An inroad from three-dimensional elasticity theory, Phys. Rev. B 97, 235102 (2018).

[56] M. Pretko and R. M. Nandkishore, Localization of extended quantum objects, Phys. Rev. B 98, 134301 (2018).

[57] D. J. Williamson, Z. Bi, and M. Cheng, Fractonic matter in symmetry-enriched $u(1)$ gauge theory, Phys. Rev. B 100, 125150 (2019).

[58] A. Dua, D. J. Williamson, J. Haah, and M. Cheng, Compactifying fracton stabilizer models, Phys. Rev. B 99, 245135 (2019).

[59] B. Shi and Y.-M. Lu, Deciphering the nonlocal entanglement entropy of fracton topological orders, Phys. Rev. B 97, 144106 (2018).

[60] H. Song, A. Prem, S.-J. Huang, and M. A. Martin-Delgado, Twisted fracton models in three dimensions, Phys. Rev. B 99, 155118 (2019).

[61] H. Ma and M. Pretko, Higher-rank deconfined quantum criticality at the lifshitz transition and the exciton bose condensate, Phys. Rev. B 98, 125105 (2018).

[62] J. Wang, K. Xu, and S.-T. Yau, Higher-rank non-Abelian tensor field theory: Higher-moment or subdimensional polynomial global symmetry, algebraic variety, Noether's theorem, and gauge, arXiv:1911.01804 [hep-th]. 
[63] D. Doshi and A. Gromov, Vortices and Fractons, arXiv:2005.03015 [cond-mat.str-el].

[64] J. Wang and S.-T. Yau, Non-Abelian gauged fractonic matter field theory: New sigma models, superfluids and vortices, arXiv:1912.13485 [cond-mat.str-el].

[65] P. Hořava, Membranes at quantum criticality, J. High Energy Phys. 03 (2009) 020.

[66] P. Hořava, Quantum gravity at a lifshitz point, Phys. Rev. D 79, 084008 (2009).

[67] C. Xu and P. Hořava, Emergent gravity at a lifshitz point from a bose liquid on the lattice, Phys. Rev. D 81, 104033 (2010).
[68] H. P. Büchler, M. Hermele, S. D. Huber, M. P. A. Fisher, and P. Zoller, Atomic Quantum Simulator for Lattice Gauge Theories and Ring Exchange Models, Phys. Rev. Lett. 95, 040402 (2005).

[69] B. Paredes and I. Bloch, Minimum instances of topological matter in an optical plaquette, Phys. Rev. A 77, 023603 (2008).

[70] N. Seiberg, Field theories with a vector global symmetry, SciPost Phys. 8, 050 (2020).

[71] A. Paramekanti, L. Balents, and M. P. A. Fisher, Ring exchange, the exciton bose liquid, and bosonization in two dimensions, Phys. Rev. B 66, 054526 (2002). 